\title{
CONFORMAL INVARIANTS OF SUBMANIFOLDS
}

\author{
CHUAN-CHIH HSIUNG ${ }^{1}$ AND LARRY R. MUGRIDGE
}

\begin{abstract}
A local conformal invariant and a global conformal invariant of a submanifold immersed in a Euclidean space are derived.
\end{abstract}

1. Introduction. It is well known (Haantjes [3]) that every conformal mapping $f$ on a Euclidean $m$-space $E^{m}$ can be decomposed into a product of similarity transformations (i.e., Euclidean motions and homotheties) and inversions $\left\{\pi_{i}\right\}$. Let $x: M^{n} \rightarrow E^{m}$ be an $n$-dimensional submanifold immersed in $E^{m}$. For simplicity we shall write $x\left(M^{n}\right)$ as $M^{n}$. A quantity on $M^{n}$ is a conformal invariant if it is invariant under the conformal mappings of $E^{m}$, for which the center of every inversion does not lie on $M^{n}$.

Let $e$ be a unit normal vector of $M^{n}$ at a point $x$. Then the first fundamental form of $M^{n}$ at $x$ and the second fundamental form of $M^{n}$ at $x$ with respect to $e$ are respectively defined to be

$$
\mathrm{I}=d x \cdot d x, \quad \mathrm{II}\left(e_{x}\right)=-d x \cdot d e,
$$

where $d x$ and $d e$ are vector-valued linear forms on $M^{n}$, and the dot denotes the inner product of two vectors in $E^{m}$; actually the form $I$ is the Riemannian metric on $M^{n}$ induced by the immersion. The eigenvalues $h_{1}(e), \ldots, h_{n}(e)$ of II $(e)$ relative to I are called the principal curvatures of $M^{n}$ at the point $x$ with respect to $e$, and the $r$ th mean curvature of $M^{n}$ at $x$ with respect to $e$ is defined to be the $r$ th elementary symmetric function of $h_{1}(e), \ldots, h_{n}(e)$ divided by the number of terms, i.e.,

$$
\left(\begin{array}{l}
n \\
r
\end{array}\right) H_{r}(e)=\sum_{i_{1}, \ldots i_{r}=1}^{n} h_{i_{1}}(e) \ldots h_{i_{r}}(e), \quad r=1, \ldots, n,
$$

where $\left(\begin{array}{l}n \\ r\end{array}\right)$ is the binomial coefficient.

Let $B$ be the bundle of unit normal vectors of $M^{n}$, so that a point of $B$ is a pair $(x, e)$. Then $B$ is a bundle of $(m-n-1)$-dimensional spheres $S^{m-n-1}$ of unit normal vectors over $M^{n}$ and is a manifold of dimension $m-1$. Let $d V_{n}$ and $d \sigma_{m-n-1}$ be the volume elements of $M^{n}$ and $S^{m-n-1}$ at a point $x$ respectively.

The purpose of this paper is to establish the following theorem.

\section{THEOREM.}

Received by the editors October 17, 1975.

AMS (MOS) subject classifications (1970). Primary 53B25; Secondary 53A30, 53C40.

Key words and phrases. Conformal mappings, inversions, mean curvatures, conformal invariants.

${ }^{1}$ The work of this author was partially supported by NSF Grant GP-43665. 


$$
K:=\left\{\int_{S^{m-n-1}}\left[H_{1}(e)^{2}-H_{2}(e)\right]^{n / 2} d \sigma_{m-n-1}\right\} d V_{n}
$$

is a local conformal invariant of the submanifold $M^{n}$ immersed in $E^{m}$, and

$$
\int_{M^{n}} K
$$

is a global conformal invariant of a compact oriented $M^{n}$ in $E^{m}$.

This Theorem is due to W. Blaschke [1] for $m=3, n=2$, and due to B. Y. Chen [2] for $n=2$ and a general $m$. Moreover, for a compact oriented $M^{2}$, by using the well-known Gauss-Bonnet formula from (1.4), it follows that

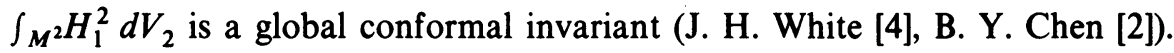

2. Proof of the Theorem. It is obvious that $K$ is invariant under similarity transformations, so that it suffices to show that $K$ is invariant under an inversion $\pi$ on $E^{m}$, whose center does not lie on the submanifold $M^{n}$.

Choose the center of the inversion $\pi$ to be the origin of a coordinate system in the Euclidean space $E^{m}$, and let $x, \bar{x}$ be the position vectors of a pair of corresponding points of the submanifold $M^{n}$ and its image submanifold $\bar{M}^{n}$ under $\pi$. Then the definition of an inversion implies

$$
\bar{x}=\left(c^{2} r^{-2}\right) x, \quad r^{2}=x \cdot x,
$$

where $c$ is the radius of the inversion $\pi$. By (2.1) we readily obtain

$$
\begin{gathered}
d \bar{x}=\left(c^{2} r^{-2}\right) d x-2\left(c^{2} r^{-3} d r\right) x, \\
d \bar{x} \cdot d \bar{x}=\left(c^{4} r^{-4}\right) d x \cdot d x .
\end{gathered}
$$

Let $e_{n+1}, \ldots, e_{m}$ be any $m-n$ mutually orthogonal unit normal vectors of $M^{n}$ at $x$. Then from (2.2) it is easy to see that

$$
\bar{e}_{\alpha}=2 r^{-2}\left(x \cdot e_{\alpha}\right) x-e_{\alpha}, \quad \alpha=n+1, \ldots, m,
$$

are $m-n$ mutually orthogonal unit normal vectors of $\bar{M}^{n}$ at $\bar{x}$. Similarly, if $e$ is a general unit normal vector of $M^{n}$ at $x$, then

$$
\bar{e}=2 r^{-2}(x \cdot e) x-e
$$

is a unit normal vector of $\bar{M}^{n}$ at $\bar{x}$. Since $e$ can be written as

$$
e=\sum_{\alpha=n+1}^{m} a_{\alpha} e_{\alpha}, \quad \sum_{\alpha=n+1}^{m} a_{\alpha}^{2}=1
$$

we have

$$
\bar{e}=\sum_{\alpha=n+1}^{m} a_{\alpha} \bar{e}_{\alpha} .
$$

Thus, if the vector $e$ moves over the sphere $S^{m-n-1}$ of $M^{n}$ at $x$, then the vector $\bar{e}$ moves over the $(m-n-1)$-dimensional sphere $\bar{S}^{m-n-1}$ of unit normal vectors of $\bar{M}^{n}$ at $\bar{x}$.

By means of (2.2) and (2.5) we obtain 


$$
d \bar{x} \cdot d \bar{e}=2 c^{2} r^{-4}(x \cdot e) d x \cdot d x-\left(c^{2} r^{-2}\right) d x \cdot d e,
$$

and therefore, in consequence of (2.3),

$$
d \bar{x} \cdot d \bar{e}+\lambda d \bar{x} \cdot d \bar{x}=-\frac{c^{2}}{r^{2}}\left[d x \cdot d e+\left(-\frac{2(x \cdot e)}{r^{2}}-\lambda \frac{c^{2}}{r^{2}}\right) d x \cdot d x\right] .
$$

Let $d \bar{V}_{n}$ be the volume element of the submanifold $\bar{M}^{n}$ at a point $\bar{x}$, and $\bar{h}_{1}(\bar{e}), \ldots, \bar{h}_{n}(\bar{e})$ be the principal curvatures of $\bar{M}^{n}$ at $\bar{x}$ with respect to $\bar{e}$. From (2.3), (2.9) and (1.1) it follows that

$$
\begin{gathered}
d \bar{V}_{n}=(c / r)^{2 n} d V_{n}, \\
\bar{h}_{i}(e)=-c^{-2} r^{2} h_{i}(e)-2 c^{-2}(x \cdot e), \quad i=1, \ldots, n .
\end{gathered}
$$

Thus by (1.2) and its corresponding equation for $\bar{M}^{n}$ we have

$$
\begin{gathered}
\bar{H}_{1}(\bar{e})=-c^{-2} r^{2} H_{1}(e)-2 c^{-2}(x \cdot e), \\
\bar{H}_{2}(\bar{e})=c^{-4} r^{4} H_{2}(e)+4 c^{-4} r^{2}(x \cdot e) H_{1}(e)+4 c^{-4}(x \cdot e)^{2},
\end{gathered}
$$

which, together with (2.10), immediately imply

$$
\left[\bar{H}_{1}(\bar{e})^{2}-\bar{H}_{2}(\bar{e})\right]^{n / 2} d \bar{V}_{n}=\left[H_{1}(e)^{2}-H_{2}(e)\right]^{n / 2} d V_{n} \text {. }
$$

Integrating both sides of (2.14) and using (1.3) we obtain where $\bar{K}$ is defined by

$$
\bar{K}=\left\{\int_{\bar{S}^{m-n-1}}\left[\bar{H}_{1}(e)^{2}-\bar{H}_{2}(e)\right]^{n / 2} d \bar{\sigma}_{m-n-1}\right\} d V_{n},
$$

$d \sigma_{m-n-1}$ being the volume element of $S^{m-n-1}$ at $x$. If $M^{n}$ is compact and oriented, then by integrating both sides of (2.15) over $M^{n}$ we have

$$
\int_{M^{n}} \bar{K}=\int_{M^{n}} K
$$

Hence our Theorem is proved.

It should be noted that a hypersphere of $E^{m}$ has vanishing invariant $K$ since the principal curvature $h_{1}(e), \ldots, h_{n}(e)$ of the hypersphere with respect to the unique unit normal vector $e$ at every point $x$ is equal.

\section{REFERENCES}

1. W. Blaschke, Vorlesungen über Differentialgeometrie. III, Springer, Berlin, 1929.

2. B. Y. Chen, An invariant of conformal mappings, Proc. Amer. Math. Soc. 40 (1973), 563-564. MR 47 \#9489.

3. J. Haantjes, Conformal representations of an $n$-dimensional euclidean space with a non-definite fundamental form on itself, Nederl. Akad. Wetensch. Proc. Ser. A 40 (1937), 700-705.

4. J. H. White, A global invariant of conformal mappings in space, Proc. Amer. Math. Soc. 38 (1973), 162-164. MR 48 \#2954.

Department of Mathematics, Lehigh University, Bethlehem, Pennsylvania 18015

Department of Mathematics, Kutztown State College, Kutztown, Pennsylvania 19530 